BULL. AUSTRAL. MATH. SOC.

VOL. 23 (1981); 395-412.

\title{
DEGREE OF APPROXIMATION OF A FUNCTION BY NÖRLUND MEANS OF ITS FOURIER SERIES
}

\author{
R.B. SAXENA
}

Communicated by K.C. Shrivastava

Two theorems of T.M. Flett [Quart. J. Math. Oxford Ser. (2) 7 (1956), 81-95] on the degree of approximation to a function by the Cesàro means of its Fourier series are extended to Nörlund means. Their conjugate analogues are also proved.

1.

Let $f(x)$ be Lebesgue integrable and periodic with period $2 \pi$, and let

(1.1) $f(x) \sim \frac{1}{2} a_{0}+\sum_{k=1}^{\infty}\left(a_{k} \cdot \cos k x+b_{k} \cdot \sin k x\right)=\sum_{k=0}^{\infty} A_{k}(x)$

be its Fourier series.

The conjugate Fourier series of (1.1) is

$$
\sum_{k=1}^{\infty}\left(b_{k} \cdot \cos k x-a_{k} \cdot \sin k x\right)=\sum_{k=1}^{\infty} B_{k}(x) .
$$

The Nörlund mean of an infinite series $\sum_{k=1}^{\infty} a_{k}$, with the sequence of partial sums $\left\{s_{n}\right\}$, is defined (Nörlund [4], Woronoi [5]) by the sequence-

Received 18 December 1980. The author wishes to express his best thanks to $\mathrm{Dr}$ K.C. Shrivastava for the guidance and help during the preparation of this paper. The author is extremely thankful to the referee for his suggestions in the text of the paper.

395 
to-sequence transformation

$$
t_{n}\left(p_{n}\right)=\frac{1}{P_{n}} \sum_{k=0}^{n} p_{n-k} \cdot s_{k}
$$

where $\left\{p_{n}\right\}$ is a sequence of non-negative strictly monotonic decreasing constants, and

$$
P_{n}=\sum_{k=0}^{n} p_{k} \neq 0, P_{-1}=p_{-1}=0
$$

We use the following notation:

$$
\begin{aligned}
\phi(t) & =\Phi_{x}(t)=f(x+t)+f(x-t)-2 f(x), \\
\psi(t) & =\psi_{x}(t)=f(x+t)-f(x-t), \\
N_{n}\left(p_{n} ; t\right) & =\frac{1}{2 \pi P_{n}} \sum_{k=0}^{n} \frac{\sin \left(k+\frac{1}{2}\right) t}{\sin t / 2}, \\
\bar{N}_{n}\left(p_{n} ; t\right) & =\frac{1}{2 \pi P_{n}} \sum_{k=0}^{n} p_{n-k} \frac{\cos \left(k+\frac{1}{2}\right) t}{\sin t / 2}, \\
f(x) & =\frac{1}{2 \pi} \int_{0}^{\pi} \psi(t) \cot t / 2 d t, \\
\Phi_{r}(t) & =\frac{1}{\Gamma(r)} \int_{0}^{t}(t-u)^{r-1}{ }_{\phi(u) d u,}, r>0, \\
\Phi_{0}(t) & =\phi(t), \Phi_{r}(t)=\Phi_{1+r}^{\prime}(t) \quad(-1<r<0), \\
\Psi_{r}(t) & =\frac{1}{\Gamma(r)} \int_{0}^{t}(t-u)^{r-1} \psi(u) d u, r>0, \\
\Psi_{0}(t) & =\psi(t), \Psi_{r}(t)=\Psi_{1+r}^{\prime}(t) \quad(-1<r<0) .
\end{aligned}
$$

$[x]$ denotes the largest integer less than or equal to $x$.

2.

Flett [2] has proved the following theorems for the degree of approximation to a function by Cesàro means of its Fourier series. 
THEOREM A. Suppose that $f$ is integrable in $(-\pi, \pi)$ and of class Lip $\alpha$ in the closed interval $(a, b)$ where $0<\alpha<1$ and that $a_{n}, b_{n}=o\left(n^{-\beta}\right)$. If $0 \leq \beta<\alpha$ and $k \geq \alpha-\beta$, then

$$
\sigma_{n}^{k}(x)-f(x)=o\left(n^{-\alpha}\right)
$$

$\sigma_{n}^{k}(x)$ being the $(C, k)$ mean of series (1.1).

THEOREM B. Let $0<\alpha<1,0 \leq \beta<1,-1<r<0,0<\delta \leq \pi$, $k \geq \alpha-\beta, k>\alpha+r$, and let $x$ be a point such that

$$
\begin{aligned}
& \text { (i) } A_{n}(x)=O\left(n^{-\beta}\right) \text {, } \\
& \text { (ii) } \Phi_{1+p}(+0)=0 \text {, and } \int_{0}^{t} u^{-r}\left|\Phi_{1+r}(u)\right| \leq A t^{1+\alpha} \quad(0 \leq t \leq \delta), \\
& \text { and } \\
& \text { (iii) } \int_{0}^{t} \phi(u) d u=o\left(t^{-1+\alpha}\right) \text {; }
\end{aligned}
$$

then

$$
\sigma_{n}^{k}(x)-f(x)=o\left(n^{-\alpha}\right)
$$

In the present paper we generalise the above theorems for Nörlund means and also prove their conjugate analogues. Precisely we prove the following theorems.

THEOREM 1. Stppose that $f$ is integrable in $(-\pi, \pi)$ and of class Lip $\alpha$ in the closed interval $(a, b)$ where $0<\alpha<1$, and that

$$
a_{n}, b_{n}=o\left\{\left(a_{n} / Q_{n}\right)^{\beta}\right\} \text {. }
$$

If $0<\beta<\alpha$ and $\left\{p_{n}\right\},\left\{q_{n}\right\}$ and $\left\{r_{n}\right\}$ be monotonic decreasing sequences of non-negative constants such that

$$
r_{n} / R_{n-1} \geq p_{n} / P_{n-1}-q_{n} / Q_{n-1}=P_{n} / P_{n-1}-Q_{n} / Q_{n-1},
$$

$Q_{n}$ and $R_{n}$ being defined similarly to $P_{n}$, then 


$$
\left.t_{n}\left(r_{n}\right)-f(x)=o\left\{\left(R_{\left[P_{n} / p_{n}\right]}\right]_{n}\right) \cdot\left(p_{n} / P_{n}\right)^{\alpha}\right\} \cong o\left\{\left(p_{n} / P_{n}\right)^{\alpha}\right\} \text {, }
$$

where $t_{n}\left(r_{n}\right)$ is the Nörlund mean (1.3) of series (1.1) generated by the sequence $\left\{r_{n}\right\}$.

THEOREM 2. Suppose that $f$ is integrable in $(-\pi, \pi)$ and of class Lip $\alpha$ in the closed interval $(a, b), 0<\alpha<1$ and that

$$
a_{n}, b_{n}=o\left\{\left(q_{n} / Q_{n}\right)^{\beta}\right\} \text {. }
$$

If $0<\beta<\alpha$ and $\left\{p_{n}\right\},\left\{q_{n}\right\}$ and $\left\{r_{n}\right\}$ are sequences as defined in Theorem 1, then

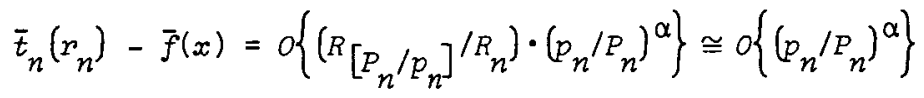

provided that the conjugate function exists, $\bar{t}_{n} \cdot\left(r_{n}\right)$ being the Norrlund mean (1.3) of series (1.2) generated by sequence $\left\{r_{n}\right\}$.

THEOREM 3. Let $0<\alpha<1,0 \leq \beta<1,-1<r<0,0<\delta \leq \pi$ and let $x$ be a point such that

$$
A_{n}(x)=o\left\{\left(q_{n} / Q_{n}\right)^{\beta}\right\} \text {, }
$$

(2.3) $\Phi_{1+r}(+0)=0$ and $\int_{0}^{t} u^{-r}\left|d \Phi_{1+r}(u)\right| \leq A t^{1+\alpha} \quad(0 \leq t \leq \delta)$,

(2.4) $\int_{0}^{t} \phi(u) d u=o\left(t^{1+\alpha}\right)$;

then

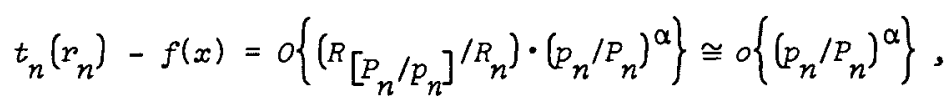

where $\left\{p_{n}\right\},\left\{q_{n}\right\}$ and $\left\{r_{n}\right\}$ are sequences as defined in Theorem 1 .

THEOREM 4. Let $0<\alpha<1,0 \leq \beta<1,-1<r<0,0<\delta \leq \pi$ and let $x$ be a point such that

$$
B_{n}(x)=o\left\{\left(q_{n} / Q_{n}\right)^{\beta}\right\} \text {, }
$$


(2.6) $\Psi_{1+r}(+0)=0$ and $\int_{0}^{t} u^{-r}\left|d \Psi_{1+r}(u)\right| \leq A^{t 1+\alpha} \quad(0 \leq t \leq \delta)$,

(2.7) $\int_{0}^{t} \psi(u) d u=o\left(t^{1+\alpha}\right)$

then

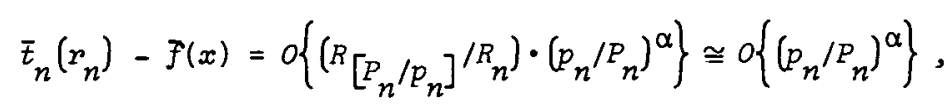

provided that the conjugate function exists, $\left\{p_{n}\right\},\left\{q_{n}\right\}$ and $\left\{r_{n}\right\}$ being sequences as defined in Theorem 1.

3.

We shall need the following lemmas in the proof of our theorems.

LEMMA 3.1. FOr $0 \leq t \leq p_{n} / P_{n}$,

$$
N_{n}\left(p_{n} ; t\right)=O(n) \text {. }
$$

Proof.

$$
\begin{aligned}
N_{n}\left(p_{n} ; t\right) & =\frac{1}{2 \pi P_{n}} \sum_{k=0}^{n} p_{n-k} \frac{\sin \left(k+\frac{1}{2}\right) t}{\sin t / 2} \\
& \leq \frac{1}{2 \pi P_{n}} \sum_{k=0}^{n} p_{n-k}(2 k+1) \\
& \leq \frac{1}{2 \pi P_{n}}(2 n+1) \sum_{k=0}^{n} p_{n-k} \\
& =O(n) .
\end{aligned}
$$

LEMMA 3.2 [3]. If the sequence $\left\{p_{n}\right\}$ is non-negative and nonincreasing then for $0 \leq a \leq b \leq \infty, 0 \leq t \leq \pi$ and any $n$, we have

$$
\left|\sum_{k=a}^{b} p_{k} e^{i(n-k) t}\right| \leq P_{\tau}
$$

for any $a$, where $\tau=\left[t^{-1}\right]$.

LEMMA 3.3. For $0<p_{\dot{n}^{\prime}} / P_{n} \leq t \leq \delta \leq \pi$, 
400

R.B. Saxena

(i) $\left|N_{n}\left(p_{n} ; t\right)\right|=O\left\{P_{\tau} / t P_{n}\right\}$,

(ii) $\left|\bar{N}_{n}\left(p_{n} ; t\right)\right|=O\left\{P_{\tau} / t P_{n}\right\}$.

Proof. (i)

$$
\begin{aligned}
\left|N_{n}\left(p_{n} ; t\right)\right| & =\frac{1}{2 \pi P_{n}}\left|\sum_{k=0}^{n} p_{k} \frac{\sin \left(n-k+\frac{3}{2}\right) t}{\sin t / 2}\right| \\
& \leq \frac{1}{2 \pi P_{n}}\left\{\left|\sum_{k=0}^{n} p_{k} \sin (n-k) t \cot t / 2\right|+\left|\sum_{k=0}^{n} p_{k} \cos (n-k) t\right|\right\} \\
& =0\left\{\left(P_{\tau} \cot t / 2\right) / P_{n}\right\}+0\left\{P_{\tau} / P_{n}\right\} \text { using Lemma } 3.2 \\
& =0\left\{P_{\tau} / t P_{n}\right\} .
\end{aligned}
$$

(ii) The estimate for $\bar{N}_{n}\left(p_{n} ; t\right)$ can be proved similarly.

LEMMA 3.4. For $0 \leq u \leq p_{n} / P_{n}$,

(i)

$$
\begin{aligned}
& J\left(p_{n} ; u\right)=\int_{p_{n} / P_{n}}^{\delta}(t-u)^{-r-1} N_{n}\left(r_{n} ; t\right) d t \\
& =o\left\{\left(R\left[_{n} / p_{n}\right]^{/ R}\right) \cdot\left(P_{n} / p_{n}\right)^{r+1}\right\} \text {; }
\end{aligned}
$$

(ii)

$$
\bar{J}\left(p_{n} ; u\right)=\int_{p_{n} / P_{n}}^{\delta}(t-u)^{-r-1} \bar{N}_{n}\left(r_{n} ; t\right) d t=o\left\{\left(R_{\left.\left.\left[P_{n} / p_{n}\right]^{/ R}\right) \cdot\left(P_{n} / p_{n}\right)^{r+1}\right\}} .\right.\right.
$$

Proof. (i)

$$
\begin{aligned}
& J\left(p_{n} ; u\right)=\int_{p_{n} / P_{n}}^{\delta}(t-u)^{-r-1} N_{n}\left(r_{n} ; t\right) d t \\
& =\int_{p_{n} / p_{n}}^{\delta}(t-u)^{-r-1} O\left\{R_{\tau} / t R_{n}\right\} d t \text { using Lemma } 3.3(i) \\
& \leq o\left\{\left(R\left[P_{n} / p_{n}\right] / R_{n}\right) \cdot\left(P_{n} / p_{n}\right)\right\} \int_{p_{n} / P_{n}}^{\delta}(t-u)^{-r-1} d t
\end{aligned}
$$

https://doi.org/10.1017/S0004972700007279 Published online by Cambridge University Press 


$$
\begin{aligned}
& =o\left\{\left(R\left[P_{n} / p_{n}\right]^{/ R}\right) \cdot\left(P_{n} / p_{n}\right)\right\} \cdot\left\{\frac{(t-u)^{-r}}{-r}\right\}_{p_{n} / P_{n}}^{\delta} \\
& =O\left\{\left(R_{\left[P_{n} / p_{n}\right]} / R_{n}\right) \cdot\left(P_{n} / p_{n}\right)\right\} \cdot\left\{\frac{(\delta-u)^{-r}}{-r}+\frac{1}{r}\left(\frac{p_{n}}{P_{n}}-u\right\}^{-r}\right\} \\
& \leq\left\{\left\{\left(R\left[P_{n} / p_{n}\right]^{/ R}\right) \cdot\left(P_{n} / p_{n}\right)^{r+1}\right\} \text { for } 0 \leq u \leq p_{n} / P_{n}\right. \text {. }
\end{aligned}
$$

(ii) The estimate for $\bar{J}\left(p_{n} ; u\right)$ can be proved similarly.

LEMMA 3.5. (i)

$$
K(u)=\int_{u}^{\delta}(t-u)^{-r-1} N_{n}\left(r_{n} ; t\right)=o\left\{u^{-1-r} \frac{R_{[1 / u]}}{R_{n}}\right\} .
$$

(ii)

$$
\bar{K}(u)=\int_{u}^{\delta}(t-u)^{-r-1} \bar{N}_{n}\left(r_{n} ; t\right)=o\left\{u^{-1-r} \frac{R[1 / u]}{R_{n}}\right\} .
$$

Proof. (i)

$$
\begin{aligned}
K(u) & =\int_{u}^{\delta}(t-u)^{-r-1} N_{n}\left(r_{n} ; t\right) d t \\
& =\int_{u}^{\delta}(t-u)^{-r-1} o\left\{R_{[\tau]} / t R_{n}\right\} d t \text { using Lemma } 3.3(i) \\
& =0\left\{\frac{[1 / u]}{u R_{n}}\right\} \cdot \int_{u}^{\delta}(t-u)^{-r-1} d t \\
& =\left\{\frac{\int^{R}[1 / u]}{u R_{n}}\right\} \cdot\left\{\frac{(t-u)^{-r}}{-r}\right\}_{u}^{\delta} \\
& =0\left\{\frac{[1 / u]}{u R_{n}}\right\} \cdot\left\{\frac{(\delta-u)^{-r}}{-r}\right\}(-1<r<0) \\
& =O\left\{\frac{[1 / u]}{u R_{n}} \cdot u^{-1-r}\right\} .
\end{aligned}
$$

(ii) The estimate for $\bar{K}(u)$ can be obtained in a similar manner.

4.

Proof of Theorem 1. Let us write (Zygmund [6]) 


$$
S_{n}(x)=\frac{3}{2} a_{0}+\sum_{k=1}^{n} A_{k}(x)
$$

then we have

$$
S_{n}(x)-f(x)=\frac{1}{2 \pi} \int_{0}^{\pi} \phi(t) \frac{\sin \left(n+\frac{1}{2}\right) t}{\sin t / 2} d t,
$$

using (1.3) for $\sum A_{k}(x)$, we have

$$
\begin{aligned}
& t_{n}\left(r_{n}\right)-f(x)=\frac{1}{R_{n}} \sum_{k=0}^{n} r_{n-k} S_{k}(x)-f(x) \\
& =\int_{0}^{\pi} \phi(t) \frac{1}{2 \pi R} \sum_{n=0}^{n} r_{n-k} \frac{\sin \left(k+\frac{1}{2}\right) t}{\sin t / 2} d t \\
& =\int_{0}^{\pi} \phi(t) N_{n}\left(r_{n} ; t\right) d t \\
& =\left\{\int_{0}^{p_{n} / P_{n}}+\int_{P_{n} / P_{n}}^{\delta}+\int_{\delta}^{\pi}\right\} \phi(t) N_{n}\left(r_{n} ; t\right) d t \\
& =I_{1}+I_{2}+I_{3} \text {, say. }
\end{aligned}
$$

Now

$$
\begin{aligned}
\left|I_{1}\right| & \leq O(n) \int_{0}^{p_{n} / P_{n}}|\phi(t)| d t \text { using Lemma } 3.1 \\
& =O(n) \cdot O\left(p_{n} / P_{n}\right)^{\alpha+1} \\
& =O\left\{\left(p_{n} / P_{n}\right)^{\alpha}\right\} \text { as } n p_{n} \leq P_{n} .
\end{aligned}
$$

Further

$(4.3)$

$$
\begin{aligned}
\left|I_{2}\right| & \leq \int_{p_{n} / P_{n}}^{\delta}|\phi(t)| \cdot\left|N_{n}\left(r_{n} ; t\right)\right| d t \\
& =\int_{p_{n} / P_{n}}^{\delta} O\left(t^{\alpha}\right) \cdot O \frac{R_{\tau}}{t R_{n}} d t \text { using Lemma } 3.3(i) \\
& =\frac{1}{R_{n}} \int_{p_{n} / P_{n}}^{\delta} O\left(t^{\left.\alpha-I_{R_{\tau}}\right) d t}\right.
\end{aligned}
$$




$$
\begin{aligned}
& \text { Approximation by Nörlund means }
\end{aligned}
$$

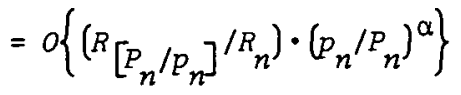

$$
\begin{aligned}
& \cong o\left\{\left(p_{n} / P_{n}\right)^{\alpha}\right\} \text {. }
\end{aligned}
$$

Now we have

$$
\phi_{x}(t) \sim A_{0}+2 \sum_{k=1}^{\infty} A_{k}(x) \cos k t
$$

where $A_{0}=a_{0}-2 f(x)$. Hence we have

(4.4) $\left|I_{3}\right| \leq \int_{\delta}^{\pi}\left|A_{0} N_{n}\left(r_{n} ; t\right)\right| d t+2 \int_{\delta}^{\pi}\left|\sum_{k=1}^{\infty} A_{k}(x) \cos k t\right| \cdot\left|N_{n}\left(r_{n} ; t\right)\right| d t$

$$
\begin{aligned}
\leq & \int_{\delta}^{\pi}\left|A_{0} N_{n}\left(r_{n} ; t\right)\right| d t+2 \int_{\delta}^{\pi}\left|\sum_{k=1}^{\infty} a_{k} \cos k x \cos k t\right| \\
& \cdot\left|N_{n}\left(r_{n} ; t\right)\right| d t+2 \int_{\delta}^{\pi}\left|\sum_{k=1}^{\infty} b_{k} \sin k x \cos k t\right| \cdot\left|N_{n}\left(r_{n} ; t\right)\right| d t \\
= & I_{3.1}+I_{3.2}+I_{3.3} \text {, say. }
\end{aligned}
$$

Now

$$
\begin{aligned}
I_{3.1} & =\int_{\delta}^{\pi}\left|A_{0} \frac{R_{\tau}}{t R_{n}}\right| d t \text { by using Lemma } 3.3(i) \\
& =O\left(1 / R_{n}\right) \\
& =O(1) \text {, as } n \rightarrow \infty .
\end{aligned}
$$

Further

$2 \sum_{k=1}^{\infty} a_{k}(x) \cos k x \cos k t=\sum_{k=1}^{\infty} 0\left\{\left(q_{k} / Q_{k}\right)^{\beta}\right\} \cdot\{\cos k(x+t)+\cos k(x-t)\}$

using (2.1)

$$
\begin{aligned}
& =\sum_{k=1}^{\infty} O(1) \cdot\{\cos k(x+t)+\cos k(x-t)\} \\
& =O(1) .
\end{aligned}
$$

Thus 
$(4.6)$

$$
\begin{aligned}
I_{3.2} & =O(1) \cdot \int_{\delta}^{\pi}\left|N_{n}\left(r_{n} ; t\right)\right| d t \\
& =O(1) \int_{\delta}^{\pi}\left|R_{\tau} / t R_{n}\right| d t \text { by Lemma } 3.3(i) \\
& =O\left(1 / R_{n}\right) \\
& =O(1), \text { as } n \rightarrow \infty .
\end{aligned}
$$

Similarly,

$(4.7)$

$$
I_{3.3}=o(1) \text {. }
$$

In view of $(4.4),(4.5),(4.6)$ and $(4.7)$,

$$
\left|I_{3}\right|=O(1) \text {. }
$$

Finally considering (4.1), (4.2), (4.3) and (4.8), the proof of the theorem is complete.

5.

Proof of Theorem 2. We have

$$
\bar{S}_{n}(x)=\frac{1}{2 \pi} \int_{0}^{\pi} \psi(t) \frac{\cos t / 2-\cos \left(n+\frac{1}{2}\right) t}{\sin t / 2} d t
$$

Using (1.3) for $\sum B_{n}(x)$, we have

$$
\text { (5.1) } \begin{aligned}
z_{n}\left(r_{n}\right)-f(x) & =\frac{1}{R_{n}} \sum_{k=0}^{n} r_{k} \bar{S}_{n-k}(x)-\frac{1}{2 \pi} \int_{0}^{\pi} \psi(t) \cot t / 2 d t \\
& =\frac{1}{R_{n}} \sum_{k=0}^{n} r_{k} \frac{1}{2 \pi} \int_{0}^{\pi} \psi(t) \frac{\cos t / 2-\cos \left(n-k+\frac{3}{2}\right) t}{\sin t / 2} d t \\
& =-\int_{0}^{\pi} \frac{1}{2 \pi R_{n}} \psi(t) \int_{k=0}^{\pi} \psi(t) \cot t / 2 d t \\
& =-\int_{0}^{\pi} \psi(t) \bar{N}_{n}\left(r_{n} ; t\right) d t
\end{aligned}
$$




$$
\begin{aligned}
& =-\left\{\int_{0}^{p_{n} / P_{n}}+\int_{p_{n} / P_{n}}^{\delta}+\int_{\delta}^{\pi}\right\} \psi(t) \bar{N}_{n}\left(r_{n} ; t\right) d t \\
& =\bar{I}_{1}+\bar{I}_{2}+\bar{I}_{3} \text {, say. }
\end{aligned}
$$

Since the conjugate function exists, we have

$$
\frac{1}{2 \pi} \int_{0}^{p_{n} / P} \psi(t) \cot t / 2 d t=o(1) .
$$

Hence

$$
\begin{aligned}
& \frac{1}{2 \pi} \int_{0}^{p_{n} / P} n \psi(t) \cot t / 2 d t+\bar{I}_{1} \\
& =\frac{1}{2 \pi} \int_{0}^{p_{n} / P_{n}} \psi(t)\left\{\cot t / 2-\frac{1}{R_{n}} \sum_{k=0}^{n} \frac{r_{k} \cos \left(n-k+\frac{1}{2}\right) t}{\sin t / 2}\right\} d t \\
& =\frac{1}{2 \pi R_{n}} \int_{0}^{p_{n} / P_{n}} \psi(t) \sum_{k=0}^{n} r_{k}\left\{\sum_{\nu=0}^{n-k} 2 \sin v t\right\} d t \\
& \leq \frac{1}{2 \pi R_{n}} \int_{0}^{p_{n} / P_{n}}|\psi(t)| \sum_{k=0}^{n} n r_{k} d t \\
& =o(n) \int_{0}^{p_{n} / P_{n}} o\left(t^{\alpha}\right) d t \\
& \cong o\left\{\left(p_{n} / P_{n}\right)^{\alpha}\right\} \text {. }
\end{aligned}
$$

Thus

$$
\left|\bar{I}_{1}\right|=o\left\{\left(p_{n} / P_{n}\right)^{\alpha}\right\}+o(1)
$$

Further 
$(5.3)$

$$
\begin{aligned}
& \left|\bar{I}_{2}\right|=\int_{p_{n} / P_{n}}^{\delta}|\psi(t)| \cdot\left|\bar{N}_{n}\left(r_{n} ; t\right)\right| d t \\
& =\int_{p_{n} / P_{n}}^{\delta}|\psi(t)| \cdot O\left(R_{\mathrm{T}} / t R_{n}\right) d t \text { using Lemma } 3.3(i i) \\
& =o\left(I / R_{n}\right) \int_{P_{n} / P_{n}}^{\delta} O\left(t^{\alpha-1}\right) \cdot O\left(R_{\tau}\right) d t
\end{aligned}
$$

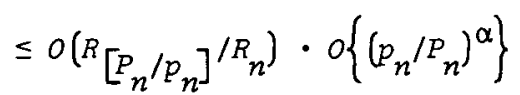

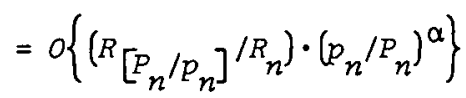

$$
\begin{aligned}
& \cong o\left\{\left(p_{n} / P_{n}\right)^{\alpha}\right\} \text {. }
\end{aligned}
$$

Finally, since

$$
\psi(t) \sim 2 \sum_{k=1}^{\infty} B_{k}(x) \sin k t,
$$

we have

$$
\text { (5.4) } \begin{aligned}
\left|\bar{I}_{3}\right| & \leq 2 \int_{\delta}^{\pi}\left|\sum_{k=1}^{\infty} B_{k}(x) \sin k t\right|\left|\bar{N}_{n}\left(r_{n} ; t\right)\right| d t \\
& \leq 2 \int_{\delta}^{\pi}\left|\sum_{k=1}^{\infty} b_{k} \cos k x \sin k t\right|\left|\bar{N}_{n}\left(r_{n} ; t\right)\right| d t \\
& \quad+2 \int_{\delta}^{\pi}\left|\sum_{k=1}^{\infty} a_{k} \sin k x \sin k t\right|\left|\bar{N}_{n}\left(r_{n} ; t\right)\right| d t \\
& =\bar{I}_{3.1}+\bar{I}_{3.2}, \text { say. }
\end{aligned}
$$

Now

$$
\begin{aligned}
2 \sum_{k=1}^{\infty} b_{k} \cos k x \sin k t & =\sum_{k=1}^{\infty} 0\left\{\left(q_{k} / Q_{k}\right)^{\beta}\right\} \cdot\{\sin k(x+t)-\sin k(x-t)\} \\
& \leq \sum_{k=1}^{\infty} O(1)\{\sin k(x+t)-\sin k(x-t)\} \\
& =O(1) .
\end{aligned}
$$

Therefore, we have 


$$
\begin{aligned}
\bar{I}_{3.1} & =O(1) \int_{\delta}^{\pi}\left|\bar{N}_{n}\left(r_{n} ; t\right)\right| d t \\
& =O(1) \int_{\delta}^{\pi} O\left\{R_{\tau} / t R_{n}\right\} d t \text { by using Lemma } 3.3(i i) \\
& =O\left(1 / R_{n}\right) \\
& =o(1), \text { as } n \rightarrow \infty .
\end{aligned}
$$

Similarly

$$
\bar{I}_{3.2}=o(1)
$$

Considering $(5.4),(5.5)$ and $(5.6)$, we get

$$
\left|\bar{I}_{3}\right|=o(1) \text {. }
$$

Consequently in view of (5.1), (5.2), (5.3) and (5.7) the proof of Theorem 2 is complete.

6.

Proof of Theorem 3. We have, as in Theorem 1,

$$
\text { (6.1) } \begin{aligned}
t_{n}\left(r_{n}\right)-f(x) & =\left\{\int_{0}^{p_{n} / P_{n}}+\int_{p_{n} / P_{n}}^{\delta}+\int_{\delta}^{\pi}\right\} \phi(t) \cdot N_{n}\left(r_{n} ; t\right) d t \\
& =J_{1}+J_{2}+J_{3} \text {, say. }
\end{aligned}
$$

By using Lemma 3.1 , we get

$$
\begin{aligned}
J_{1} & \leq \int_{0}^{p_{n} / P_{n}} \phi(t) \cdot O(n) d t \\
& =O(n) \cdot o\left\{\left(p_{n} / P_{n}\right)^{1+\alpha}\right\} \text { by using (2.4) } \\
& =o\left\{\left(p_{n} / P_{n}\right)^{\alpha}\right\} \text { since } n p_{n} \leq P_{n} .
\end{aligned}
$$

Further, since

$$
\phi(t) \sim A_{0}+2 \sum_{k=1}^{\infty} A_{k}(x) \cos k t,
$$

where $A_{0}=a_{0}-2 f(x)$, we have 
(6.3) $\left|J_{3}\right| \leq \int_{\delta}^{\pi}\left|A_{0} N_{n}\left(r_{n} ; t\right)\right| d t+2 \int_{\delta}^{\pi}\left|\sum_{k=1}^{\infty} A_{k}(x) \cos k t\right| \cdot\left|N_{n}\left(r_{n} ; t\right)\right| d t$ $=J_{3.1}+J_{3.2}$, say.

Now

$$
J_{3.1}=o(1)
$$

as in Theorem 1. Further

$$
\begin{aligned}
\sum_{k=1}^{\infty} A_{k}(x) \cdot \cos k t \cdot N_{n}\left(r_{n} ; t\right) & =N_{n}\left(r_{n} ; t\right) \sum_{k=1}^{\infty} o\left\{\left(q_{k} / Q_{k}\right)^{\beta}\right\} \cdot \cos k t \\
& \leq 0\left\{N_{n}\left(r_{n} ; t\right)\right\} \cdot \sum_{k=1}^{\infty}\{\cos k t\} \\
& =0\left\{N_{n}\left(r_{n} ; t\right)\right\} .
\end{aligned}
$$

Thus

$$
\begin{aligned}
J_{3.2} & =\int_{\delta}^{\pi} O\left\{N_{n}\left(r_{n} ; t\right)\right\} d t \\
& =\int_{\delta}^{\pi} O\left(R_{\mathrm{T}} / t R_{n}\right) d t \text { by using Lemma } 3.3(i i) \\
& =O\left(1 / R_{n}\right) \\
& =o(1), \text { as } n \rightarrow \infty .
\end{aligned}
$$

Considering $(6.3),(6.4)$ and $(6.5)$, we have

$$
\left|J_{3}\right|=o(1) \text {. }
$$

Finally, following Bosanquet [1], we get

(6.7) $J_{2}=\frac{1}{\Gamma(-r)} \int_{p_{n} / P_{n}}^{\delta} N_{n}\left(r_{n} ; t\right) d t \int_{0}^{t}(t-u)^{-r-1} d \Phi_{r^{+}+1}(u)$

$$
\begin{aligned}
=\frac{1}{\Gamma(-r)}\left\{\int_{0}^{p_{n} / P_{n}} d \Phi_{r+1}(u) \int_{p_{n} / P_{n}}^{\delta}(t-u)^{-r-1} N_{n}\left(r_{n} ; t\right) d t\right. \\
\left.+\int_{P_{n} / P_{n}}^{\delta} d \Phi_{r+1}(u) \int_{u}^{\delta}(t-u)^{-r-1} N_{n}\left(r_{n} ; t\right) d t\right\}
\end{aligned}
$$




$$
\begin{aligned}
& =\frac{1}{\Gamma(-r)}\left\{\int_{0}^{p_{n} / P_{n}} J\left(p_{n}, u\right) d \Phi_{r+1}(u)+\int_{p_{n} / P_{n}}^{\delta} K(u) d \Phi_{r+1}(u)\right\} \\
& =J_{2.1}+J_{2.2} \text {, say. }
\end{aligned}
$$

Using Lemma 3.4 (i), we get

(6.8) J J.1 $\leq \frac{1}{\Gamma(-r)} \int_{0}^{p_{n} / P_{n}} o\left\{\left(R_{\left[P_{n} / p_{n}\right]} / R_{n}\right) \cdot\left(P_{n} / p_{n}\right)^{p+1}\right\}\left|d \Phi_{r+1}(u)\right|$

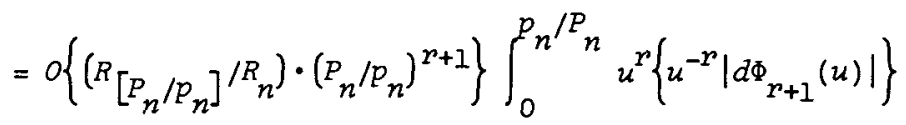

$$
\begin{aligned}
& \left.=o\left\{\left(R_{\left[P_{n} / p_{n}\right]}\right]_{n}\right) \cdot\left(P_{n} / p_{n}\right)^{r+1}\right\} \\
& \text { - }\left[\left\{u^{r} \cdot o\left(u^{1+\alpha}\right)\right\}_{0}^{p_{n} / P_{n}}-\int_{0}^{p_{n} / P_{n}} r \cdot u^{r-1} \cdot o\left(u^{1+\alpha}\right) d u\right] \\
& \text { by partial integration and using (2.3) }
\end{aligned}
$$

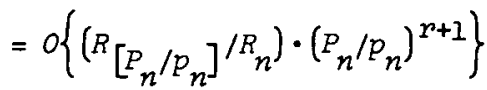

$$
\begin{aligned}
& \text { - }\left[o\left\{\left(p_{n} / P_{n}\right)^{\alpha+r+1}\right\}-r\left\{o\left(p_{n} / P_{n}\right)^{\alpha+r+1}\right\}\right]
\end{aligned}
$$

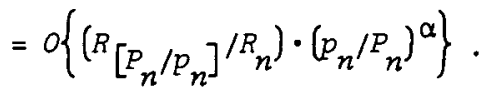

Similarly, using Lemma 3.5 (i) we get

$$
\begin{aligned}
& J_{2.2} \leq O\left(1 / R_{n}\right) \int_{p_{n} / P_{n}}^{\delta} u^{-1-r_{R_{[}}}{ }_{[1 / u]}\left|d \Phi_{r+1}(u)\right| \\
& \leq \mathcal{O}\left\{\left(R_{n}\left[P_{n}\right]^{/ p_{n}}\right) \cdot\left(P_{n} / p_{n}\right)\right\} \int_{p_{n} / P_{n}}^{\delta} u^{-r}\left|d \Phi_{r+1}(u)\right| \\
& =o\left\{\left(R_{\left[P_{n} / P_{n}\right]} / R_{n}\right) \cdot\left(p_{n} / P_{n}\right)^{\alpha}\right\} \text { by using (2.3). }
\end{aligned}
$$

From (6.7), (6.8) and (6.9), we get 


$$
\begin{aligned}
& J_{2}=o\left\{\left(R_{\left|P_{n} / p_{n}\right|} / R_{n}\right) \cdot\left(p_{n} / P_{n}\right)^{\alpha}\right\} \\
& \cong o\left\{\left(p_{n} / P_{n}\right)^{\alpha}\right\} \text {. }
\end{aligned}
$$

By virtue of $(6.1),(6.2),(6.6)$ and $(6.10)$, the proof of Theorem 3 is complete.

\section{7.}

Proof of Theorem 4. We have, as in Theorem 2,

$$
\begin{aligned}
Z_{n}\left(r_{n}\right)-f(x) & =-\left\{\int_{0}^{p_{n} / P_{n}}+\int_{p_{n} / P_{n}}^{\delta}+\int_{\delta}^{\pi}\right\} \psi(t) \bar{N}_{n}\left(r_{n} ; t\right) d t \\
& =\bar{J}_{1}+\bar{J}_{2}+\bar{J}_{3}, \text { say. }
\end{aligned}
$$

Proceeding as in Theorem 2, we get

$$
\bar{J}_{1}=o\left\{\left(p_{n} / P_{n}\right)^{\alpha}\right\}+o(1) \text {. }
$$

Further, we write as in Theorem 2,

$$
\psi(t) \sim 2 \sum_{k=1}^{\infty} B_{k}(x) \sin k t
$$

whence we have

$$
\left|\bar{J}_{3}\right| \leq 2 \cdot \int_{\delta}^{\pi}\left|\sum_{k=1}^{\infty} B_{k}(x) \sin k t\right|\left|\bar{N}_{n}\left(r_{n} ; t\right)\right| d t .
$$

Now

$$
\begin{aligned}
\sum_{k=1}^{\infty} B_{k}(x) \sin k t & =\sum_{k=1}^{\infty} O\left(q_{k} / Q_{k}\right)^{B} \sin k t \text { by using (2.5) } \\
& =O(1) \sum_{k=1}^{\infty} \sin k t \\
& =O(1) .
\end{aligned}
$$

Thus 


$$
\begin{aligned}
\left|\bar{J}_{3}\right| & \leq o(1) \int_{\delta}^{\pi}\left|\bar{N}_{n}\left(r_{n} ; t\right)\right| d t \\
& =o\left(1 / R_{n}\right) \text { by using Lemma } 3.3(i i) \\
& =o(1), \text { as } n \rightarrow \infty .
\end{aligned}
$$

Now, using the fractional integration for $\psi(t)$, we get

$$
\text { (7.4) } \begin{aligned}
\bar{J}_{2}= & \frac{1}{\Gamma(-r)} \int_{p_{n} / P_{n}}^{\delta} \bar{N}_{n}\left(r_{n} ; t\right) \int_{0}^{t}(t-u)^{-r-1} d \Psi \Psi_{r+1}(u) \\
= & \frac{1}{\Gamma(-r)}\left\{\int_{0}^{p_{n} / P_{n}} d \Psi_{r+1}(u) \int_{p_{n} / P_{n}}^{\delta}(t-u)^{-r-1} \bar{N}_{n}\left(r_{n} ; t\right) d t\right. \\
& \left.+\int_{p_{n} / P_{n}}^{\delta} d \Psi_{r+1}(u) \int_{u}^{\delta}(t-u)^{-r-1} \bar{N}_{n}\left(r_{n} ; t\right) d t\right\}
\end{aligned}
$$

by changing the order of integration

$$
\begin{aligned}
& =\frac{1}{\Gamma(-r)}\left\{\int_{0}^{p_{n} / P_{n}} \bar{J}\left(p_{n}, u\right) d \Psi_{r+1}(u)+\int_{p_{n} / P_{n}}^{\delta} \bar{K}(u) d \Psi_{r+1}(u)\right\} \\
& =\bar{J}_{2.1}+\bar{J}_{2.2} \text {, say. }
\end{aligned}
$$

Using Lemma 3.4 (ii) and 3.5 (ii) and (2.6) and proceeding similarly as in Theorem 3, we get

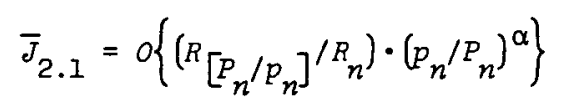

and

$$
\bar{J}_{2.2}=o\left\{\left(R_{\left.\left.\left[P_{n} / p_{n}\right]^{/ R_{n}}\right) \cdot\left(p_{n} / P_{n}\right)^{\alpha}\right\}}\right.\right.
$$

From $(7.4),(7.5)$ and $(7.6)$ we get

$$
\bar{J}_{2}=o\left\{\left(R_{\left.\left.\left[P_{n} / p_{n}\right]^{/ R}\right) \cdot\left(p_{n} / p_{n}\right)^{\alpha}\right\}}\right.\right.
$$

Consequently, in view of $(7.1),(7.2),(7.3)$ and $(7.7)$, the proof of Theorem 4 is complete. 


\section{References}

[1] L.S. Bosanquet, "Some extensions of Young's criterion for the convergence of a Fourier series", Quart. J. Math. Oxford Ser. 6 (1935), 113-123.

[2] T.M. Flett, "On the degree of approximation to a function by the Cesàro means of its Fourier series", Quart. J. Math. Oxford Ser. (2) 7 (1956), 81-95.

[3] Leonard McFadden, "Absolute Nörlund summability", Duke Math. J. 9 (1942), 168-207.

[4] N.E. Nörlund, "Sur une application des functions permutables", Acta Univ. Lund. (2) 16 (1919), Nr. 8.

[5] G.F. Woronoi, "Extension of the notion of the limit of the sum of terms of an infinite series", Ann. of Math. (2) 33 (1932), $422-428$.

[6] Antoni Zygmund, Trigonometrical series (Monografje Matematiyczne, 5. Z Subwencji Funduszu Kultury Narodowej, Warszawa, Lwow, 1935).

\footnotetext{
Department of Mathematics and Statistics,

University of Saugar,

Sagar (M.P.),

India 470003.
} 